\title{
XOR-Forwarding for Wireless Networks
}

\author{
Hsiang-Po Wang*, Yi-Ta Chuang*, Chih-Wei Yi*, Yu-Chee Tseng* and Pin-Chuan Liu ${ }^{\dagger}$ \\ ${ }^{*}$ Department of Computer Science, National Chiao Tung University \\ 1001 University Road, Hsinchu City 30010, Taiwan \\ Emails: kingpo.cs96g@g2.nctu.edu.tw, \{izek.cs96g,yi,yctseng\}@nctu.edu.tw \\ ${ }^{\dagger}$ Industrial Technology Research Institute, Hsinchu, Taiwan, R.O.C. \\ Email: flash@itri.org.tw
}

\begin{abstract}
In this paper, we propose a localized networkcoding-based packet forwarding protocol for wireless networks, called Opportunistic ASynchronous Information diSsemination (OASIS), based on COPE. OASIS not only inherents two features from COPE, opportunistic listening and opportunistic coding, but also introduces a new one, opportunistic information dissemination, which aggressively encodes as many packets as possible even if packets are not going to be received by their next hops. In addition, we point out that packet pool managements and packet information exchanges are critical in the implementation. Simulation results show that OASIS has network throughput about 1.4 times of traditional unicast forwarding, and the improvement is about 1.2 times of the improvement achieved by COPE.
\end{abstract}

Index Terms-Network coding, wireless networks, OASIS, COPE

\section{INTRODUCTION}

Wireless networks providing a ubiquitous mobile computing environment play an important role in today's modern life. However, the capacity of wireless networks is limited by available radio frequency bandwidth, and what's worse, wireless communications suffer the problem of radio frequency interference that further reduces the network capacity especially in a dense random access network. Network coding is a novel idea proposed to improve multicast capacity by utilizing network topology and coding multiple information in a single packet [1]. A review of network coding and its possible Internet applications can be found in [2].

In a multi-hop network, nodes may belong to more than one routing path. Network coding suggests encoding multiple packets from different paths to form a coded packet and then multicast the coded packet. Receivers can recover the original packets by decoding coded packets received from different routing paths. In other words, network coding utilizes path diversity by intermediate node coding to increase network throughput. In order to make uses of network coding, the underlying network should be with two properties: path diversity and multicast capability. Fortunately, for wireless networks, the broadcast nature of wireless communications provide an environment to implement network coding.

Figure 1 illustrates a possible wireless network coding scenario. There are two workstations, Alice and Bob, and one relay node, Charlie. Alice and Bob need Charlie to relay messages for each other. Assume Alice has a packet $p_{1}$ for Bob, and Bob has a packet $p_{2}$ for Alice. One possible

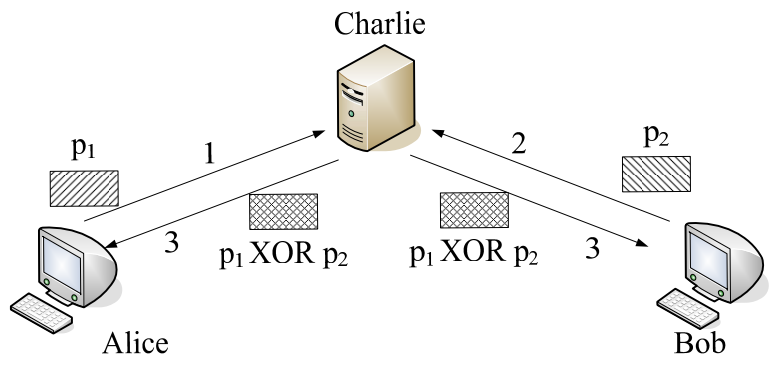

Fig. 1. Alice and Bob scenario

transmission schedule is like this: Alice and Bob respectively transmit $p_{1}$ and $p_{2}$ to Charlie, and then Charlie relays $p_{1}$ to Bob and $p_{2}$ to Alice. It takes 4 time slots to complete the task. However, there is a smart way for Charlie to relay packets: After Charlie receives $p_{1}$ and $p_{2}$, instead of unicasting $p_{1}$ and $p_{2}$ sequentially, he broadcasts $p_{1} \oplus p_{2}$. Since Alice knows $p_{1}$, she can decode $p_{2}$ by calculating $p_{1} \oplus\left(p_{1} \oplus p_{2}\right)$. Similarly, since Bob knows $p_{2}$, he can decode $p_{1}$ by calculating $p_{2} \oplus\left(p_{1} \oplus p_{2}\right)$. So, totally only 3 time slots are needed, and the latency is reduced by $1 / 4$. Eventually, network throughput is improved. Actually, such scenarios can be found frequently in wireless networks, e.g., WiFi networks, mesh networks, ad hoc networks. The best part is that such an idea can be implemented in a localized and distributed manner. This is an important edge for wireless ad hoc networks.

Katti et al. [3] proposed a localized wireless network coding heuristic, called COPE, that adopt the idea illustrated in the previous example. In COPE, nodes store overheard or relayed packets and they exchange the list of possessed packets with their 1-hop neighbors via piggy-backing or beacoming. As one node wants to transmit a packet, it greedily mixes as many packets in its output buffer as possible by the XOR operation if each XORed packet is the only one unknown among all XORed packets to the packet's next hop, and then the mixed packet is broadcasted. To prevent ambiguity, in what follows, if necessary, original packets before XORed are called plain packets and mixed packets to be transmitted over wireless links are called coded packets.

In the encoding procedure of COPE, plain packets can be mixed to form a coded packet only if all plain packets can be decoded by their corresponding next-hop receivers. Behind 
COPE, there are two important tasks. One is how to collect more packets for decoding received coded packets. The other is how to exchange the lists of possessed packets with 1hop neighbors for making proper encoding decisions. COPE can be improved if we can find other means to enrich the contents of one's packet pool. Besides the original heuristics used in COPE, we propose to further encode packets that can be decoded by non-next-hop nodes such that we can let neighbors learn more packets. Meanwhile, if the space for storing packts is limited, we need to consider a practical management heuristic. We call this new feature opportunistic information dissemination which further encodes as many plain packets as possible in coded packets even if those packets are not intended by their corresponding next-hops such that in following transmissions, there is a possibility to encode more plain packets, and therefore, network throughput can be further improved. In addition, we also provide the details of packet format for neighbors to exchange the list of possessed packets.

The rest of this paper is organized as follows. In Section II, we introduce some related works. In Section III, the proposed Opportunistic ASynchronous Information diSsemination (OASIS) protocl is presented. In Section IV, practical implementation issues are discussed. In Section V, simulation results and analyses are given. Finally, conclusions and future works are given in Section VI.

\section{RELATED WORKS}

The concept of network coding was first presented in the pioneering work by Ahlswede et al. (2000) [1] in which they showed that multicast capacity can be increased by properly mixing information from different sources at intermediate nodes. Following this thought, many works on designing efficient network coding algorithms to improve network capacity were published. Li et al. (2003) [4] proposed a linear network coding scheme for multicast in direct acyclic networks that can provide a throughput for each individual destination like in the case of unicast. [5]. In [6], Koetter and Médard proposed polynomial time encoding and decoding algorithms that were then extended to random coding by Ho et al. [7].

Gkantsidis and Rodriguez [8] proposed a randomized and distributed large content distribution solution that adopted the concept of network coding and utilized network broadcasting. When a source node wants to share a large file to other nodes, the source node divides the file into equal size segments and broadcasts enough number of linearly independent combinations of these segments into the network. If one node receives enough linearly independent combinations, it can recover the original file. To increase the independence among linear combinations, instead of purely relaying received combinations, intermediate nodes may broadcast a new linear combination from received combinations. Kumar et al. [9] proposed a broadcasting scheme over erasure channels with perfect replies. They classified the packet buffer management mechanisms into two catogories: drop-when-decode and dropwhen-see. In the drop-when-decode scheme, for a specified sender, a plain packet can be dropped if and only if every receiver has decoded the packet. As for the drop-when-see scheme, a packet is seen by a receiver if this packet is a leading variable in the decoding matrix of the receiver. A plain packet can be dropped if it has been seen by all receivers such that every receiver can decode this packet in the near future. A major issue is how to choose the coefficients for encoding.

Recently, researchers focus on the benefits of implementing network coding in wireless networks. In the presence of omnidirectional antennae, the problem of minimizing communication costs was formulated as a linear programming problem and solved in a distributed manner [10]. Yang and Chen [11] [12] proved that the problem was NP-hard, gave a lower bound of the optimal throughput, and also derived a throughput upper bound of the algorithms proposed by them. Katti et al. (2006) [3] proposed a network coding technique for wireless mesh networks, named COPE, that was implemented as a protocol layer inserted between the IP and MAC layers. COPE introduced two features of wireless network coding:

- Opportunistic listening: Due to the broadcast nature of wireless communications, nodes can overhear packets sent by their neighbors. Nodes store overheard and relayed packets and use them to decode coded packets.

- Opportunistic coding: Nodes learn the information of what packets their neighbors stored by means of, e.g. periodical information exchanges. While sending packets, nodes use this information to select plain packets for encoding such that receivers can decode the coded packet.

In this paper, we adopt these two features from COPE and further introduce a new one. We have two observations from COPE. First, COPE always encodes packets for corresponding next-hops. Second, each node maintains a packet pool to store overheard or relayed packets and uses them to decode packets. Based on the above observations, a plain packet will not be encoded in a coded packet if there is no sufficient plain packets in the corresponding next hop's packet pool. However, if we relief the limitation to let coded packets carry extra plain packets for not corresponding negihbors, we can disseminate packets to neighbors' packet pools and then increase the probability of encoding more packets in future transmissions. Therefore, we introduce a new feature, named opportunistic information dissemination, which tries to encode as many plain packets as possible even if some plain packets are not intended by the receivers.

\section{THE OASIS ALGORITHM}

OASIS is a two-phase greedy coding algorithm based on COPE. In the first phase, the algorithm encodes as many plain packets as possible into the coded packet which is similar to COPE. In the second phase, we implement the opportunistic information dissemination. Without affecting the decoding possibility of the plain packets encoded in the previous phase, this phase can enrich neighbors' packet pools and therefore is helpful for following encoding/decoding processes and potentially increases network throughput.

We first give definitions of terms used in the following sections. A plain packet is intended for (or for for short) 
a certain node if this node is the next hop in the route of this packet. Each node has one output queue that keeps all plain packets to be transmitted. For simplicity, we denote the output queue of node $R$ as queue $e^{R}$ in the following sections. Besides the output queue, each node also maitains several virtual queues each of which is a subsequence of the output queue and is corresponding to a 1-hop neighbor. A virtual queue contains all packets for its corresponding neighbor. Let virtualQueue ${ }_{w}^{R}$ denote the virtual queue of node $R$ for neighbor $w$. Each node also maintains a packet pool that stores the copy of packets this node has received, overheard or sent. The packet pool is for decoding packets. The more packets are collected in one's packet pool, the higher probability it can decode received coded packets. We use pool $^{R}$ to denote the packet pool of node $R$, and we say a node knows a packet means that this packet is in its pool. In addition, each node maintains a information database which records the lists of packets stored in each 1-hop neighbor's packet pool. We use info $o_{w}^{R}$ to denote the packet list about neighbor $w$ in $R$ 's information database. The packet lists are collected by information exchange mechanisms. As one makes encoding decisions, it needs to make sure receivers can decode the coded packets. Therefore, the information about neighbors' pools is a key information to verify whether the combination of plain packets is proper or not.

Here we give an example to illustrate the coding procedure in COPE. In the example, there are 3 nodes, $n_{1}, n_{2}$ and $n_{3}$, and a relay node $R$. $R$ has packets $p_{1}, p_{2}$, and $p_{3}$ in its output queue for $n_{1}, n_{2}$, and $n_{3}$, respectively, i.e., queue ${ }^{R}=\left(p_{1}, p_{2}, p_{3}\right)$, and virtualQueue $n_{n_{1}}^{R}=\left\{p_{1}\right\}$, virtualQueue $e_{n_{2}}^{R}=\left\{p_{2}\right\}$ and virtualQueue $n_{3}=\left\{p_{3}\right\}$. Besides, $n_{1}, n_{2}$, and $n_{3}$ know $p_{2}$, $p_{3}$, and $p_{1}$, respectively, i.e., pool ${ }^{n_{1}}=\left\{p_{2}\right\}$, pool ${ }^{n_{2}}=\left\{p_{3}\right\}$ and pool $_{3}=\left\{p_{1}\right\}$, and at the same time in $R$ 's information database, info $o_{n_{1}}^{R}=\left\{p_{2}\right\}$, info $o_{n_{2}}^{R}=\left\{p_{3}\right\}$ and info $o_{n_{3}}^{R}=$ $\left\{p_{1}\right\}$. See Fig 2. According to COPE, relay node $R$ will unicast $p_{1}$ to $n_{1}$ since $p_{1}$ is at the head of the output queue and no other plain packets can be encoded together. After $p_{1}$ is transmitted, $n_{1}$ and $n_{2}$ update their pools. Therefore, pool $^{n_{1}}=\left\{p_{1}, p_{2}\right\}$ and pool $_{2}=\left\{p_{1}, p_{3}\right\}$, info $o_{n_{1}}^{R}=\left\{p_{1}, p_{2}\right\}$ and inf $o_{n_{2}}^{R}=\left\{p_{1}, p_{3}\right\}$. Later, based on pakcet information, $p_{2}$ is unicasted to $n_{2} \cdot p_{3}$ is unicasted to $n_{3}$. Therefore, COPE executes three unicasts sequentially and needs three time slots to complete this task. There dose not exist any encoding possibility if we follow COPE. Therefore, in this case, COPE performs like the ordinary unicast.

Consider the same configuration in Fig. 2. Actually, there is a coding schedule that only needs two time slots to complete the same task. Let $R$ transmit $p_{1} \oplus p_{2}$ and $p_{2} \oplus p_{3}$ sequentially. After $p_{1} \oplus p_{2}$ is transmitted, $n_{1}$ can decode $p_{1}$ from $p_{1} \oplus p_{2}$. Although $p_{2}$ is not intended for $n_{3}, n_{3}$ can decode $p_{2}$ from $p_{1} \oplus p_{2}$ and store $p_{2}$ in its pool. In the following transmission, $p_{2}$ and $p_{3}$ can be encoded together since both $n_{2}$ and $n_{3}$ have enough information to decode $p_{2}$ and $p_{3}$, respectively. So, all $n_{1}, n_{2}$ and $n_{3}$ have the packets for them. In this case, the throughput is improved from 1 (packet/time slot) to 1.5 (packet/time slot). As we saw from the above example, if we

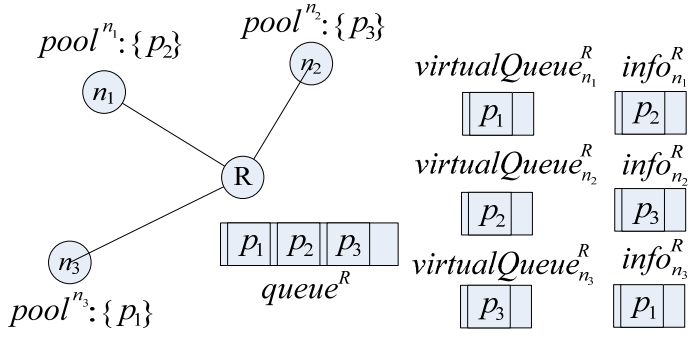

Fig. 2. An counterexample that OASIS system can have more benefit than COPE architecture

consider not only desired packets in the encoding procedure but also packets that may increase future encoding possibility, we can further increase the system throughput. OASIS aggressively encodes as many plain packets as possible. The reason for doing so is to enrich neighbors' pools such that we have higher probability to encode more packets. Below we present the encoding and decoding procedures.

\section{A. Encoding Procedure}

See PROCEDURE 1 for the psudocode of the encoding procedure. As one is going to transmit a packet, it first dequeues a (plain) packet $p$ from its output queue and assigns it to codedPacket. A set nextHop records the receivers of the coded packet and the next-hop of $p$ is the first element of the set. Let neighbor be the set of all 1-hop neighbors. Then, for each node $w$ in neighbor - nextHop, it dequeues a packet $q$ from the virtual queue virtualQueue ${ }_{w}$ and checks whether every node in nextHop $\cup\{w\}$ can decode codedPacket $\oplus q$ or not. If codedPacket $\oplus q$ can be decoded by all nodes in nextHop $\cup\{w\}$, then update nextHop and codedPacket to nextHop $\cup\{w\}$ and codedPacket $\oplus q$, respectively, and remove $q$ from queue. Otherwise, $q$ is placed back to virtualQueue $w$. The task will repeat until all neighbors are checked once. This is the first phase of the encoding procedure. The heuristic greedily encodes as many as packets in a transmission.

In the second phase, for each packet $r$ in the queue, if codedPacket $\oplus r$ can be decoded by all nodes in nextHop and some other nodes, then update codedPacket $=$ codedPacket $\oplus r$ and add those nodes to nextHop. Note that $r$ is not removed from the queue and it is possible for more than two nodes being able to decode $r$. We can greedily add the nodes in the nextHop set, however, it can be tradeoff for following unencoding possibility. In the second phase, opportunistic information dissemination is a greedy heuristic for increasing common knowledge for future transmissions.

\section{B. Decoding Procedure}

A node decodes one packet from codedPacket by the procedure given in Procedure 2. The decoding procedure is simple and similar to that in COPE. A node can decode a plain packet from codedPacket composed of $n$ plain packets 
by using the $X O R$ operation if it has all other $n-1$ plain packets in its pool. Then it checks whether this plain packet is for itself. If so, the packet is stored in the pool and forwarded to the upper layer for future processing; otherwise, the packet is stored in the pool for future decoding purposes. Note that the decoding procedure aggressively decodes coded packets and stores them in the pools if possible.

The packet pool and information database are two key components to realize either COPE or OASIS. For encoding, nodes need the information of neighbors' pools to make encoding decisions. On the other hand, for decoding, nodes need packets in their pools to decode coded packets. So we may need efficient pool managements and packet information exchanges.
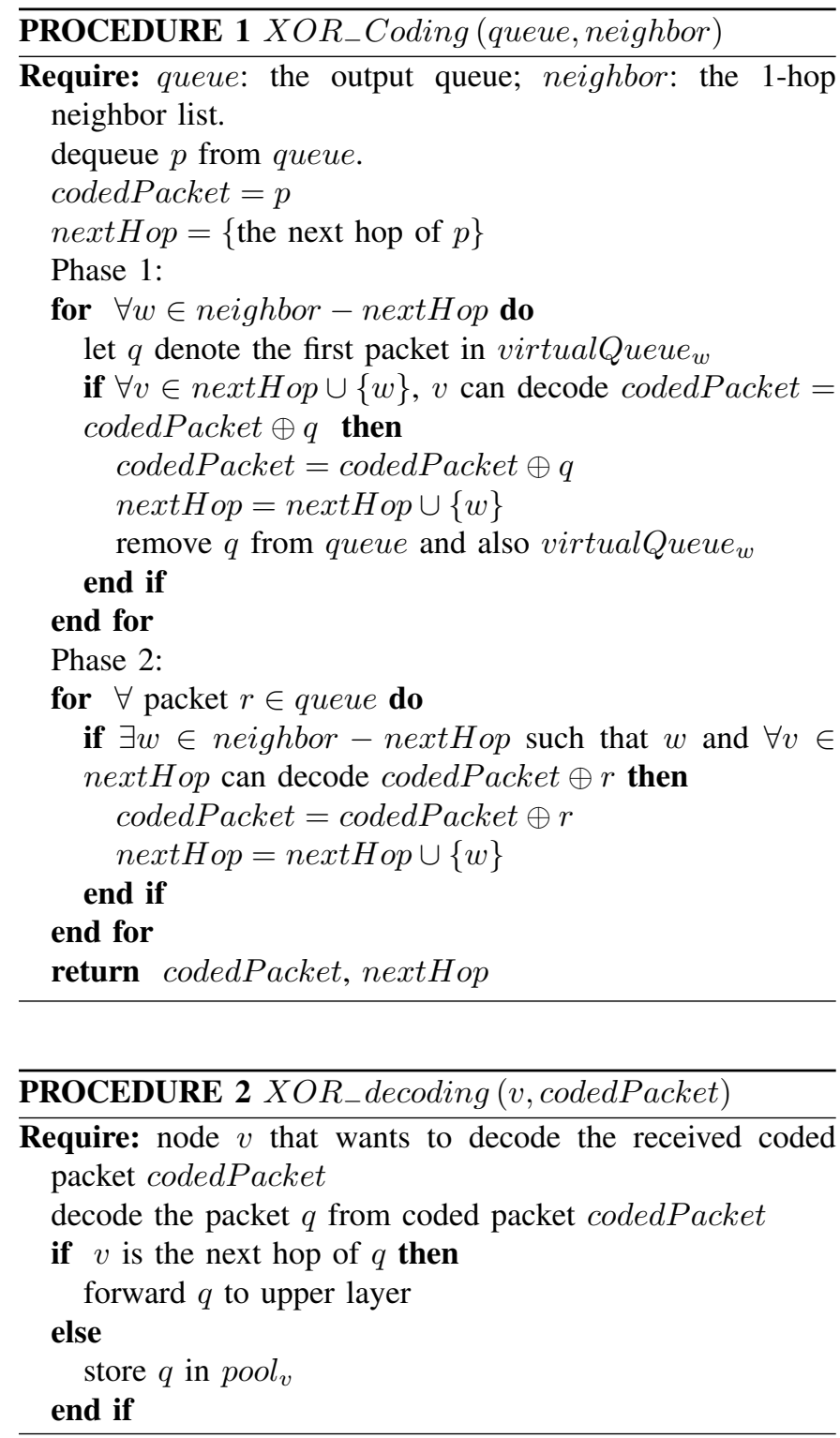

\section{PRACTICAL Issues}

This section describes some implementation details, including packet formats, packet acknowledgements and packet information exchanges. OASIS adds a variable-length header in each packet between IP and MAC headers as shown in Fig. 3. The header consists of three blocks:

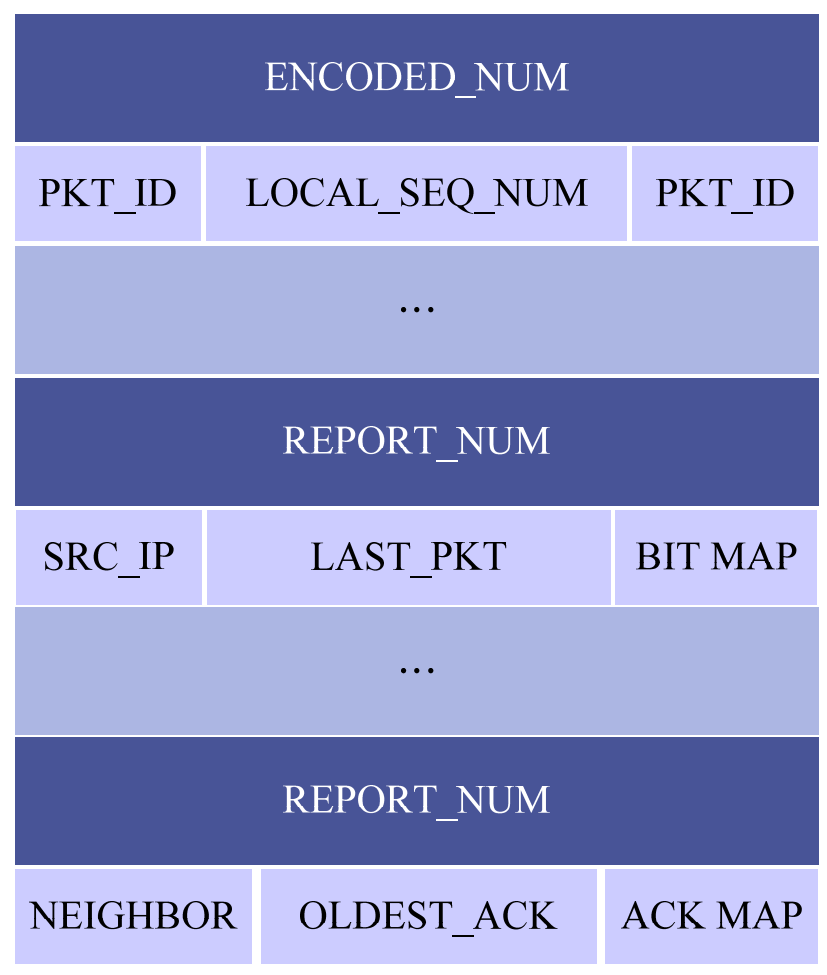

Fig. 3. OASIS's header format

- The encoded packet list: The first block records all plain packets that are encoded in this packet. It starts with ENCODED_NUM, the number of XORed plain packets, followed by a list of the XORed plain packets. The list records PKT_ID, LOCAL_SEQ_NUM and NEXTHOP for each plain packet. PKT_ID is a 32-bit hash of the packet's source IP address and IP sequence number, LOCAL_SEQ_NUM is a 16-bit counter used to identify packets between neighbors. Whenever the node sends a packet to neighbors, the counter is incremented by one and its value is assigned to LOCAL_SEQ_NUM, NEXTHOP is the MAC address of the plain packet's next hop.

- The packet pool report: The second block in the header is a list about packets stored in sender's pool. REPORT_NUM is the number of the report entries, and each entry is composed of a source IP address (SRC_IP), a IP sequence number (LAST_PKT) and a bit-map (BIT_MAP). The LAST_PKT entry indicates the last packet stored in the pool sent by SRC_IP. BIT_MAP records the recently received packets from SRC_IP. The report information is important since a node in a wireless environment needs this information to make coding decisions.

- Asynchronous acknowledgement: It is possible for more than one packet being transmitted under 
TABLE I

SIMULATION PARAMETERS

\begin{tabular}{|c|c|}
\hline Parameter type & Parameter value \\
\hline \hline Simulation duration & $1500 \mathrm{sec}$ \\
\hline Deployment region & $2000 \mathrm{~m} \times 2000 \mathrm{~m}$ \\
\hline Channel model & Accumulative noise \\
\hline SNR threshold & 10.0 \\
\hline Number of nodes & 64 \\
\hline Packet generating rate per flow & $0.019^{\sim} 0.19 \mathrm{Mbps}$ \\
\hline MAC protocol & IEEE 802.11 \\
\hline \hline Propagation path-loss model & TWO-RAY \\
\hline \hline Channel bandwidth & $1 \mathrm{Mbps}$ \\
\hline CBR data sessions & 32 \\
\hline IP output queue size & 100 packets \\
\hline Maximal retransmission times & 2 \\
\hline
\end{tabular}

network coding paradigm. However, the traditional RTS/CTS/DATA/ACK or DATA/ACK mechanism in MAC can only protect one ongoing packet. The third block in the header is therefore to acknowledge the receiving of other encoded plain packets. ACK_NUM is the number of ack entries in this packet. Each entry is composed of a neighbor MAC address (NEIGHBOR), a packet local sequence number (OLDEST_ACK) to indicate where the ack begins and a cumulative ack bit-map (ACK_MAP) to indicate previously received and missing packets by NEIGHBOR. Bit 1 indicates the packet is received and bit 0 means the packet is not yet been received and needed to be retransmitted. Note that, we ack the oldest packet that is different with COPE. To avoid redundant acks, every plain packet is acked for no more than twice.

Since the packet pool size is limited, the entry with the most reference hits is chosen to be replaced with a new entry when there are no more free entries in the packet pool. The default hit time is set to 2 .

\section{Simulation Results}

The performance of OASIS is evaluated by simulations running on GloMoSim 2.03 [13]. In the simulations, 64 nodes are depolyed in a $2000 \mathrm{~m} \times 2000 \mathrm{~m}$ square region to form a $8 \times 8$ grid. Each node has four near by nodes with distance $\frac{2000}{7} m$. An accumulated noise channel model is applied and the signalto-interference ratio (SIR) threshold in the simulation is set to 10.0. All nodes have the same transmission power with corresponding transmission range approximated to $r=376 \mathrm{~m}$. A packet will be transmitted at most 3 times before it is dropped. Note that in COPE or OASIS, a coded packet may contain more than 1 plain packet. Only the one at the head of the output buffer is responsible for the collisions and will be dropped. Each node independently generates constant bit rate (CBR) traffic into the network based on Poisson arrival with packet generating rate varied between $20 \mathrm{~ms}$ to $200 \mathrm{~ms}$. The CBR data packet size is 512 bytes. Each node randomly choose a node as its destination, and packets will be routed along the shortest hop-distance paths. The details of simulation parameters are depicted in Table I.

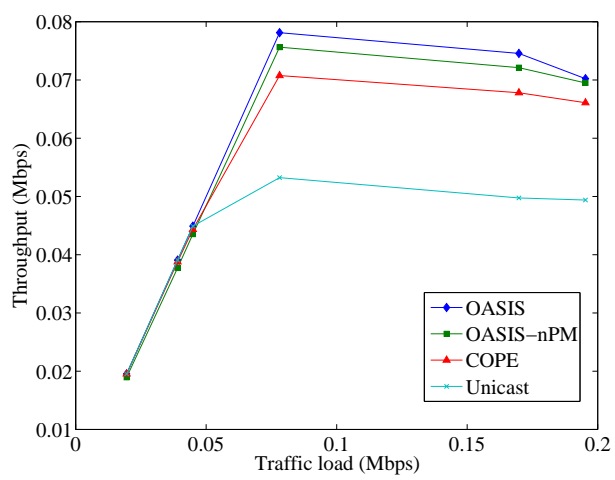

Fig. 4. Traffic load v.s. throughput in a $8 \times 8$ grid.

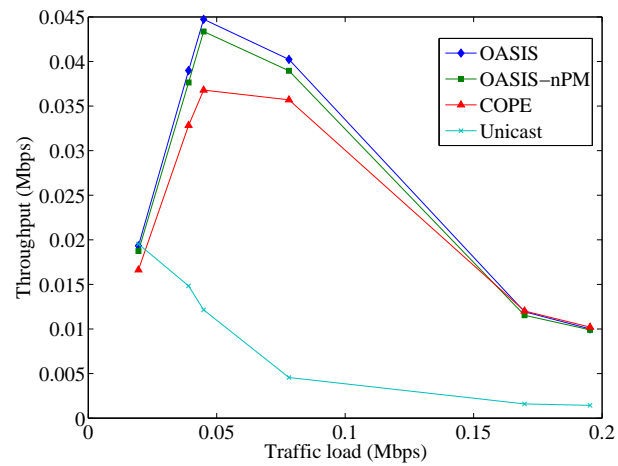

Fig. 5. Traffic load v.s. throughput in a $4 \times 4$ grid.

In the simulation, we compare the performance of classical unicast forwarding (denoted by Unicast), COPE [3] (denoted by COPE), and OASIS (denoted by OASIS). In addition, to validate the necessity of packet pool managements, we further run a version of OASIS without pool managements denoted by OASIS-nPM.

First, we measure network throughputs under various packet generation rates. The network throughput is defined as the average number of bytes received by one destination per second in the network. The classical packet generation rates v.s. network throughputs graph is depicted in Fig. 4 where $x$-axis and $y$-axis represent for packet generation rates and network throughputs in Mbps, respectively. Note that the IP queue size is 100 in the simulation. In the figure, the blue line marked with diamonds represents for the throughput for OASIS, the green line marked with squares represents for OASIS-nPM, the red line marked with triangles represents for COPE and the cyan line marked with crosses represents for Unicast.

The peak throughputs are at $(0.07,0.053),(0.16,0.065)$, $(0.16,0.071)$ and $(0.16,0.074)$ for Unicast, COPE, OASISnPM, and OASIS, respectively. Both OASIS and OASISnPM outperform COPE and Unicast. COPE, OASIS-nPM, and OASIS respectively have improvements in the maximal throughput about $122 \%, 133 \%$, and $139 \%$ compared to Uni- 


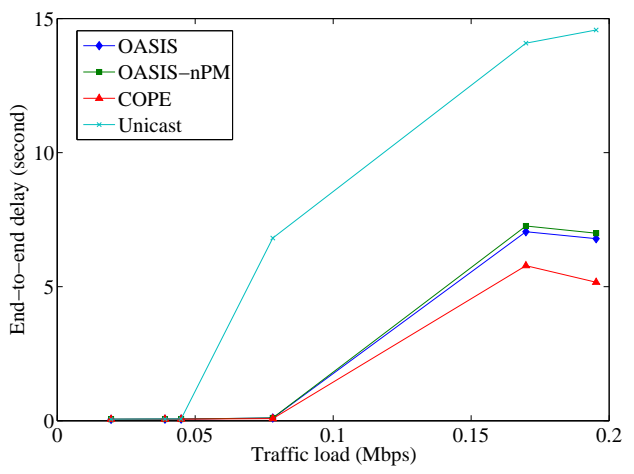

Fig. 6. Traffic load v.s. end-to-end delay in a $8 \times 8$ grid.

cast. In average, the improvements of COPE and OASIS are about $120 \%$ and $138 \%$, respectively. So, the improvements achieved by OASIS are about 1.2 times of the improvements achieved by COPE. OASIS has a superior performance result as compared with OASIS-nPM. We can see packet pool managements are necessary in network coding paradigm. In addition, we also ran a simulation with a $4 \times 4$ grid topology and the result is shown in Fig. 5.

The result is reasonable. Unicast has the worst throughput since it never utilizes broadcast property of wireless communications. In each transmission, there is at most one packet can be delivered. However, network coding schemes have the possibility to deliver more than one packet in each transmission. Moreover, OASIS and OASIS-nPM outperforms COPE since they forward extra information that is not necessary but may be helpful for future transmissions.

The end-to-end delay is defined as the total time experience as the time a packet generated from the source to the time this packet received by the destination. Figure. 6 illusrates the packet generation rate v.s. end-to-end delay with a $8 \times 8$ gird topology. We can see that the network coding schemes not only improve system capacity but also reduce end-to-end delay.

\section{Conclusions}

In this paper, we proposed a generic network coding protocol called OASIS based on COPE and introduce a new future called opportunistic information dissemination. The opportunistic information dissemination encodes as many plain packets as possible in coded packets and enriches neighbors' packet pools to increase the future encdoding possibility. Meanwhile, a simple packet pool management is introduced to deal with the limitation on the pool size. The simulation results show that OASIS has the network throughput about $139 \%$ as compared to the classical unicast forwarding. The improvement is 1.2 times than the improvement achieved by COPE. Moreover, the simulation results also show that it takes a few overhead on end-to-end delay to implement OASIS. So, overall speaking, OASIS is an offerable localized network coding heuristic for wireless networks.

In this work, we consider the implementation issues of design a network coding protocol in a wireless environment. However, there are still many things need to be considered when implement network coding into network protocol suit, such as more advance pool management mechanisms, ack mechanisms, and so on. All are possible research topics for future works.

\section{ACKNOWLEDGMENT}

We thank Prof. Chien-Chung Shen from Department of Computer Information Science, University of Delaware for his helpful comments.

This work of C.-W. Yi was supported in part by NSC under Grant No. NSC97-2221-E-009-052-MY3, by ITRI under Grant No. 8352BA4124, by MoEA under Grant No. 98-EC17-A-02-S2-0048, and by the MoE ATU plan.

\section{REFERENCES}

[1] R. Ahlswede, N. Cai, S.-Y. R. Li, and R. W. Yeung, "Network information flow," IEEE Transactions on Information Theory, vol. 46, no. 4, pp. 1204-1216, July 2000

[2] P. Chou and Y. Wu, "Network coding for the internet and wireless networks," IEEE Signal Processing Magazine, vol. 24, no. 5, pp. 77-85, September 2007.

[3] S. Katti, H. Rahul, W. Hu, D. Katabi, M. Médard, and J. Crowcroft, "XORs in the air: Practical wireless network coding," IEEE/ACM Transactions on Networking, vol. 16, no. 3, pp. 497-510, June 2008, first appeared in sigcomm 2006.

[4] S.-Y. R. Li, R. W. Yeung, and N. Cai, "Linear network coding," IEEE Transactions on Information Theory, vol. 49, no. 2, pp. 371-381, February 2003.

[5] S. Fong and R. Yeung, "Variable-rate linear network coding," in Information Theory Workshop (ITW '06), October 2006, pp. 409-412.

[6] R. Koetter and M. Médard, "An algebraic approach to network coding," IEEE/ACM Transactions on Networking, vol. 11, no. 5, pp. 782-795, Octobor 2003.

[7] T. Ho, R. Koettert, M. Médard, D. R. Karger, and M. Effros, "The benefits of coding over routing in a randomized setting," in IEEE International Symposium on Information Theory (ISIT 2003), 29 June-4 July 2003, p. 442.

[8] C. Gkantsidis and P. Rodriguez, "Network coding for large scale content distribution," in Proceedings of the 24th Annual Joint Conference of the IEEE Computer and Communications Societies (IEEE INFOCOM 2005), vol. 4, March 2005, pp. 2235-2245.

[9] J. K. Sundararajan, D. Shah, and M. Médard, "ARQ for network coding," in IEEE International Symposium on Information Theory, 2008. ISIT 2008., July 2008, pp. 1651-1655.

[10] D. Lun, N. Ratnakar, R. Koetter, M. Médard, E. Ahmed, and H. Lee, "Achieving minimum-cost multicast: a decentralized approach based on network coding," in Proceedings of the 24th Annual Joint Conference of the IEEE Computer and Communications Societies (IEEE INFOCOM 2005), vol. 3, March 2005, pp. 1607-1617.

[11] D.-N. Yang and M.-S. Chen, "On bandwidth-efficient data broadcast," IEEE Transactions on Knowledge and Data Engineering, vol. 20, no. 8, pp. 1130-1144, August 2008.

[12] _ "Data broadcast with adaptive network coding in heterogeneous wireless networks," IEEE Transactions on Mobile Computing, vol. 8, no. 1, pp. 109-125, January 2009.

[13] X. Zeng, R. Bagrodia, and M. Gerla, "GloMoSim: a library for paralle simulation of large-scale wireless networks," in Workshop on Parallel and Distributed Simulation, 1998, pp. 154-161. 